\title{
An Adaptive Scheme to Estimate Unknown Parameters of an Unmanned Aerial Vehicle
}

\author{
Imil Hamda Imran \\ Department of Engineering \\ Lancaster University \\ Bailrigg, Lancaster, LA1 4YW, United Kingdom \\ i.imran@lancaster.ac.uk
}

\author{
Allahyar Montazeri \\ Department of Engineering \\ Lancaster University \\ Bailrigg, Lancaster, LA1 4YW, United Kingdom \\ a.montazeri@lancaster.ac.uk
}

\begin{abstract}
This paper deals with tracking control problem for six degrees of freedom (6-DOF) nonlinear quadrotor unmanned aerial vehicle (UAV). A virtual control design using PD controller is proposed for tracking control position. The rotational dynamics of UAV is considered to have several unknown parameters such as propeller inertia, rotational drag coefficient and an external disturbance parameter. To handle this issue, an adaptive scheme using the certainty equivalence principle is developed. The basic idea behind this scheme is to cancel the nonlinear term by applying a similar nonlinear structure in the feedback control design. The unknown parameters are replaced by estimated parameters generated by adaptation law. The rigorous theoretical design and simulation results are presented to demonstrate the effectiveness of the controller.
\end{abstract}

Index Terms-Immersion \& invariance, unmanned aerial vehicle, 6-DOF, certainty equivalence principle, adaptive control

\section{INTRODUCTION}

Robotics and autonomous systems have recently drawn the attention of academics and industries in various fields to assist human in challenging environments such as off-shore and underwater applications, space exploration, mining and volcanic fields, and nuclear decommissioning. For example, in $[9,13]$ the use of robotic manipulator and quadcopter for the nuclear decommissioning application was investigated. Improving autonomy and cognition of robotic systems can unlock their potential for hazard monitoring. To move towards this objective, design and development of suitable controllers are an urgent need, especially for UAV applications [10]. Many control approaches have already been tested on UAVs for different scenarios. As the size of UAV is becoming smaller, the stabilization and control problems become more challenging in front of disturbances and uncertainties in real scenarios.

The UAV trajectory flight relies on four individual rotors configured in plus or cross configuration. Three degrees of freedom in UAV is related to translational motion, letting it to

The work is supported by the Engineering and Physical Sciences Research Council (EPSRC), grant number EP/R02572X/1, and National Centre for Nuclear Robotics. The authors are grateful for the support of the National Nuclear Laboratory (NNL) and the Nuclear Decommissioning Authority (NDA).

978-1-7281-8763-1/20/\$31.00 @2020 Crown have backward, forward, vertical and lateral motion. The other three states are related to rotational motions, i.e. roll, pitch and yaw. Therefore, four control inputs are available to design a controller with the capability of regulating highly coupled states of the UAV. Since regulating the position and orientation of UAV requires six states as the system output, we are dealing with an under-actuated system, which is not a straightforward problem from control system design perspective.

The presence of nonlinearities in the rotational dynamics is a common issue in designing a proper controller for UAV. Several results were reported in the literature to address the trajectory tracking problem in UAVs. One of the common approaches proposed in the literature relies on the feedback linearization technique. An interested reader is referred to $[16,18]$, for example. However, since feedback linearization relies on a perfect dynamic model of UAV, it may not provide a good solution in practical applications.

In many realistic situations, several parameters of UAV are unavailable or may be changing as time goes on. These uncertain parameters will cause more technical difficulty in designing the controller. To address this issue, two major research lines have been proposed in the literature. The first major research line is the so called fixed gain approach or robust control approach. The idea behind this technique is to design a feedback controller, dominating the uncertain dynamics of the system. As such, the uncertain nonlinearity within a particular bound can be handled by proper design of the controller. In this case, a priori information about the uncertainty bound is required for the control design task. One of the most common methods proposed in this research line is sliding mode control. The results using sliding mode control for quadcopter applications can be found in [14, 17]. To address the chattering problem in sliding mode control techniques, significant effort has been made in the literature. In the context of UAV application, the interested reader is referred to $[12,13]$.

Adaptive control technique is the second major research line to handle uncertain nonlinear dynamics, especially for the systems with unknown constant parameters. The idea behind this technique is to cancel the unknown nonlinear dynamics by estimating the unknown parameters. An estimated parameter is updated by an adaptation law until it converges to the actual 
value. A Lyapunov like function is usually utilized to design the adaptation law.

Amongst various adaptive techniques, model reference adaptive control (MRAC) is commonly used to handle unknown parameters in the system dynamics. In this scheme, a state predictor as an ideal reference model is required to estimate the unknown constant parameters of the system. Several results, relying on the use of this technique were reported in $[11,15]$. Nevertheless, the major drawback of MRAC was the fact that the stability of the closed-loop system cannot be guaranteed [1]. This issue was resolved in $L_{1}$ adaptive control technique by including a filter in the control structure [4]. This technique was proposed in [7] for a single UAV application and in [5] for a cooperative control framework. In these two results, the error between the estimated parameter and the actual value of the unknown parameter was required to synthesize the adaptation law. Consequently, this approach is not realizable for practical implementation.

Another adaptive technique, dealing with unknown constant parameters is immersion and invariance (I\&I), proposed in $[2,8]$. In this technique, the adaptive control law is designed such that the mismatch estimation error is driven to a manifold. The closed-loop system is assumed to be input to state stable (ISS) without unknown nonlinear function. Therefore the analysis is conducted to ensure the stability in front of the mismatch estimation error dynamics. The application of this scheme for under-actuated systems can be found in [3, 7]. More sophisticated results for 6-DOF UAV dynamic with unknown constant parameters was developed in [6].

In this paper, an adaptive controller for stabilization and trajectory tracking of a quadrotor UAV with unknown constant parameters in the rotational dynamics is proposed. The unknown parameters are estimated by applying the certainty equivalence principle. This scheme has two steps. First, the controller is designed for the system under an ideal situation where unknown parameters are assumed to be known. Then, the unknown parameters of the controller are replaced by their value estimated by the adaptation law in the second step. For translational dynamics, a virtual control input using PD controller is presented for position control and tracking.

The remainder of this paper is organized as follows. In Section II, the dynamics of 6 -DOF UAV is presented. Following that, the tracking control position using PD controller and attitude control design using an adaptive control are proposed in Section III. Then in Section IV, we present a simulation to demonstrate the effectiveness of our control approach. A summary and suggestion for future work are presented in Section V.

\section{SYSTEM DYNAMICS OF UAV}

In this section, we present the system dynamics of UAV. As depicted in [7], the translational dynamics is expressed by

$$
\ddot{\eta}_{1}=-g z_{e}+J_{1}\left(\eta_{2}\right) \frac{u}{m} z_{e}-\frac{k_{t}}{m} \dot{\eta}_{1},
$$

where $g, u, m, I_{R}$, and $k_{t}$ are gravity acceleration, thrust force, mass, propeller inertia and translational drag coefficient, respectively. Vector $\eta_{1}=\left[\begin{array}{lll}x & y & z\end{array}\right]^{\top}$ is a position vector and $z_{e}=\left[\begin{array}{lll}0 & 0 & 1\end{array}\right]^{\top}$. From the property of $z_{e}$, we can see that UAV is an under-actuated as the number of control input is less than the number of output. Matrix $J_{1}\left(\eta_{2}\right)$ is a transformation matrix given by

$$
J_{1}\left(\eta_{2}\right)=\left[\begin{array}{cc}
\cos \theta \cos \psi & \sin \phi \sin \theta \cos \psi-\cos \phi \sin \psi \\
\cos \theta \sin \psi & \sin \phi \sin \theta \sin \psi+\cos \phi \cos \psi \\
-\sin \theta & \sin \phi \cos \theta \\
\cos \phi \sin \theta \cos \psi+\sin \phi \sin \psi \\
\cos \phi \sin \theta \sin \psi-\sin \phi \cos \psi \\
\cos \phi \cos \theta
\end{array}\right],
$$

where $\eta_{2}=\left[\begin{array}{lll}\phi & \theta & \psi\end{array}\right]^{\top}$ is an orientation vector. By assuming $\cos \phi$ and $\cos \theta$ to be non-zero, then

$$
J_{1}^{\top}\left(\eta_{2}\right)=J_{1}^{-1}\left(\eta_{2}\right) .
$$

The rotational dynamics is represented by

$$
\begin{aligned}
\dot{\nu}_{2}= & I_{M}^{-1}\left(-\left(\nu_{2} \times I_{M} \nu_{2}\right)-I_{R}\left(\nu_{2} \times z_{e}\right) \Omega-k_{r} \nu_{2}\right. \\
& +\tau),
\end{aligned}
$$

where $\nu_{2}=\left[\begin{array}{lll}p & q & r\end{array}\right]^{\top}$ is an angular velocity vector, $k_{r}$ is rotational drag coefficient, $\tau=\left[\begin{array}{lll}\tau_{p} & \tau_{q} & \tau_{r}\end{array}\right]^{\top}$ is the torques acting in the body frame, and $I_{M}=\operatorname{diag}\left[\begin{array}{lll}I_{x} & I_{y} & I_{z}\end{array}\right]$ is an inertia matrix. The relative angular speeds of the motor $\Omega$ is computed by

$$
\Omega=\Omega_{1}-\Omega_{2}+\Omega_{3}-\Omega_{4}
$$

Both translational and rotational dynamics are highly coupling as represented by the following relationship

$$
\dot{\eta}_{1}=J_{1}\left(\eta_{2}\right) \nu_{1}, \dot{\eta}_{2}=J_{2}\left(\eta_{2}\right) \nu_{2},
$$

where $\nu_{1}=\left[\begin{array}{lll}u & v & w\end{array}\right]^{\top}$ is a linear velocity vector, and matrix $J_{2}\left(\eta_{2}\right)$ is

$$
J_{2}\left(\eta_{2}\right)=\left[\begin{array}{ccc}
1 & \sin \phi \tan \theta & \cos \phi \tan \theta \\
0 & \cos \phi & \sin \theta \\
0 & \frac{\cos \phi}{\cos \theta} & \frac{\cos \phi}{\cos \theta}
\end{array}\right] .
$$

The thrust force $u$ is generated by

$$
u=\sum_{\iota=1}^{4} f_{\iota}=\sum_{\iota=1}^{4} k_{\iota} \Omega_{\iota}^{2},
$$

where $k_{\iota}$ is a positive constant and $f_{\iota}$ is upward-lifting force generated by each rotor. The relationship between the thrust force and the torques acting around the body of UAV are expressed by the following

$$
\left[\begin{array}{c}
u \\
\tau_{p} \\
\tau_{q} \\
\tau_{r}
\end{array}\right]=\left[\begin{array}{cccc}
1 & 1 & 1 & 1 \\
l \frac{\sqrt{(2)}}{2} & -l \frac{\sqrt{(2)}}{2} & -l \frac{\sqrt{(2)}}{2} & l \frac{\sqrt{(2)}}{2} \\
l \frac{\sqrt{(2)}}{2} & l \frac{\sqrt{(2)}}{2} & -l \frac{\sqrt{(2)}}{2} & -l \frac{\sqrt{(2)}}{2} \\
d & -d & d & -d
\end{array}\right]\left[\begin{array}{l}
f_{1} \\
f_{2} \\
f_{3} \\
f_{4}
\end{array}\right]
$$


For convenience presentation, the attitude dynamics (3) with an additional external disturbance can be rewritten in the following linearly parameterized form

$$
\dot{\nu}_{2}=f\left(\nu_{2}\right) \nu_{2}+g_{1}\left(\nu_{2}\right) I_{R}+g_{2}\left(\nu_{2}\right) k_{r}+h \delta+u,
$$

where

$$
\begin{gathered}
f\left(\nu_{2}\right)=-\left[\begin{array}{ccc}
0 & \frac{I_{z}-I_{y}}{I_{x}} r & 0 \\
0 & 0 & \frac{I_{x}-I_{z}}{I_{y}} p \\
\frac{I_{y}-I_{x}}{I_{z}} q & 0 & 0
\end{array}\right] \\
g_{1}\left(\nu_{2}\right)=\left[\begin{array}{c}
\frac{-\Omega q}{I_{x}} \\
\frac{\Omega p}{I_{y}} \\
0
\end{array}\right], g_{2}\left(\nu_{2}\right)=-\left[\begin{array}{c}
\frac{p}{I_{x}} \\
\frac{q}{I_{y}} \\
\frac{r}{I_{z}}
\end{array}\right], u=I_{M}^{-1} \tau .
\end{gathered}
$$

In this paper, $I_{R}$ and $k_{r}$ are unknown for feedback control design. The external disturbance is represented by $h \delta$ that contains a known vector function of time $h$ and an unknown constant $\delta$.

\section{Proposed CONTROL DESIGN}

In this section, we present the control strategies for both translational and rotational dynamics of UAV. For translational dynamics, a virtual control using PD controller for the tracking error position in Section III-A. In another side, an adaptive control scheme is proposed for rotational dynamics with uncertain parameters. The adaptive controller is developed using the certainty equivalence principle in Section III-B.

\section{A. Translational control design}

The tracking control for translational dynamics in this section is presented from [6]. We define the tracking error of the system to be

$$
\tilde{\eta}_{1}=\eta_{1_{d}}-\eta_{1},
$$

where $\tilde{\eta}_{1}$ is the error vector position and $\eta_{1 d}$ is the desired vector position. We have the second-order dynamics of 9

$$
\ddot{\tilde{\eta}}_{1}+K_{D} \dot{\tilde{\eta}}_{1}+K_{P} \tilde{\eta}_{1} \text {. }
$$

It is easy to see that system dynamics (10) satisfies RouthHurwitz stability criterion for any positive definite $K_{P}$ and $K_{D}$. We can rewrite the dynamics (9) as

$$
\ddot{\eta}_{1}=\ddot{\eta}_{1_{d}}+K_{D}\left(\dot{\eta}_{1_{d}}-\dot{\eta}_{1}\right)+K_{P}\left(\eta_{1_{d}}-\eta_{1}\right) .
$$

Let virtual input $U=\ddot{\eta}_{1}=\left[\begin{array}{lll}U_{1} & U_{2} & U_{3}\end{array}\right]^{\top}$. By substituting $U$ to (1), then we have

$$
U=-g z_{e}+J_{1}\left(\eta_{2}\right) \frac{u}{m} z_{e}-\frac{k_{t}}{m} \dot{\eta}_{1} .
$$

By doing several mathematical calculation as presented in [6], we can compute $\phi$ and $\theta$

$$
\begin{aligned}
\phi= & \arcsin \left(( U _ { 1 } \operatorname { s i n } \psi - U _ { 2 } \operatorname { c o s } \psi ) \left(\left(U_{1}+\frac{k_{t}}{m} \dot{x}\right)^{2}\right.\right. \\
& \left.\left.+\left(U_{2}+\frac{k_{t}}{m} \dot{y}\right)^{2}+\left(U_{3}+g+\frac{k_{t}}{m} \dot{z}\right)^{2}\right)^{1 / 2}\right) \\
\theta= & \arctan \left(\frac{U_{1} \cos \psi+U_{2} \sin \psi}{U_{3}+g+\frac{k_{t}}{m}}\right),
\end{aligned}
$$

and $\phi_{d}$ and $\theta_{d}$

$$
\begin{aligned}
\phi_{d}= & \arcsin \left(( U _ { 1 } \operatorname { s i n } \psi _ { d } - U _ { 2 } \operatorname { c o s } \psi _ { d } ) \left(\left(U_{1}+\frac{k_{t}}{m} \dot{x}_{d}\right)^{2}\right.\right. \\
& \left.\left.+\left(U_{2}+\frac{k_{t}}{m} \dot{y}_{d}\right)^{2}+\left(U_{3}+g+\frac{k_{t}}{m} \dot{z}_{d}\right)^{2}\right)^{1 / 2}\right) \\
\theta_{d}= & \arctan \left(\frac{U_{1} \cos \psi_{d}+U_{2} \sin \psi_{d}}{U_{3}+g+\frac{k_{t}}{m} \dot{z}_{d}}\right)
\end{aligned}
$$

The total thrust can be computed by

$$
\begin{aligned}
u= & m\left(U_{1}(\cos \phi \sin \theta \cos \psi+\sin \phi \sin \psi)\right. \\
& +U_{2}(\cos \phi \sin \theta \sin \psi-\sin \phi \cos \psi) \\
& \left.+\left(U_{3}+g+\frac{k_{t}}{m}\right) \cos \phi \cos \theta\right) .
\end{aligned}
$$

\section{B. Rotational control design}

One of the challenge issues in designing controller for attitude dynamics is the presence of uncertain parameters. In case all parameters of the dynamics are available for feedback control design, it is easy to cancel the nonlinear dynamics by applying a simple feedback linearization approach. However, several parameters may unknown in many practical settings. In this paper, several parameters such as $I_{R}, k_{r}$ and $\delta$ are unknown for feedback control design. An adaptive control scheme is proposed to handle the uncertainties.

Before presenting our adaptive approach, let us define the desired trajectory such that mismatch between actual and desired trajectories to be

$$
e=\nu_{2}-\nu_{2_{d}}
$$

where $\nu_{2_{d}}=\left[\begin{array}{lll}p_{d} & q_{d} & r_{d}\end{array}\right]^{\top}$ is a vector of the desired trajectory.

The estimate parameters are generated by adaptation law along Lyapunov like function. The main objective of adaptation law is

$$
\lim _{t \rightarrow \infty} \tilde{I}_{R}(t), \tilde{k}_{r}(t), \tilde{\delta}(t)=0,
$$

where $\tilde{I}_{R}(t)=\hat{I}_{R}(t)-I_{R}, \tilde{k}_{r}(t)=\hat{k}_{r}(t)-k_{r}$ and $\tilde{\delta}(t)=\hat{\delta}(t)-\delta$. The proposed adaptive scheme is presented in Theorem 3.1.

Theorem 3.1: Consider the attitude dynamics (8). The controller is selected to be

$$
\begin{aligned}
\tau= & I_{M}\left(-\alpha e-f\left(\nu_{2}\right) \nu_{2}-g_{1}\left(\nu_{2}\right) \hat{I}_{R}-g_{2}\left(\nu_{2}\right) \hat{k}_{r}\right. \\
& -h \hat{\delta})+\dot{\nu}_{2_{d}},
\end{aligned}
$$

where $\hat{I}_{R}, \hat{k}_{r}, \hat{\delta}$ is generated by

$$
\begin{aligned}
\dot{\hat{I}}_{R} & =\gamma_{1} e^{\top} g_{1}\left(\nu_{2}\right), \\
\dot{\hat{k}}_{r} & =\gamma_{2} e^{\top} g_{2}\left(\nu_{2}\right), \\
\dot{\hat{\delta}} & =\gamma_{3} e^{\top} h,
\end{aligned}
$$




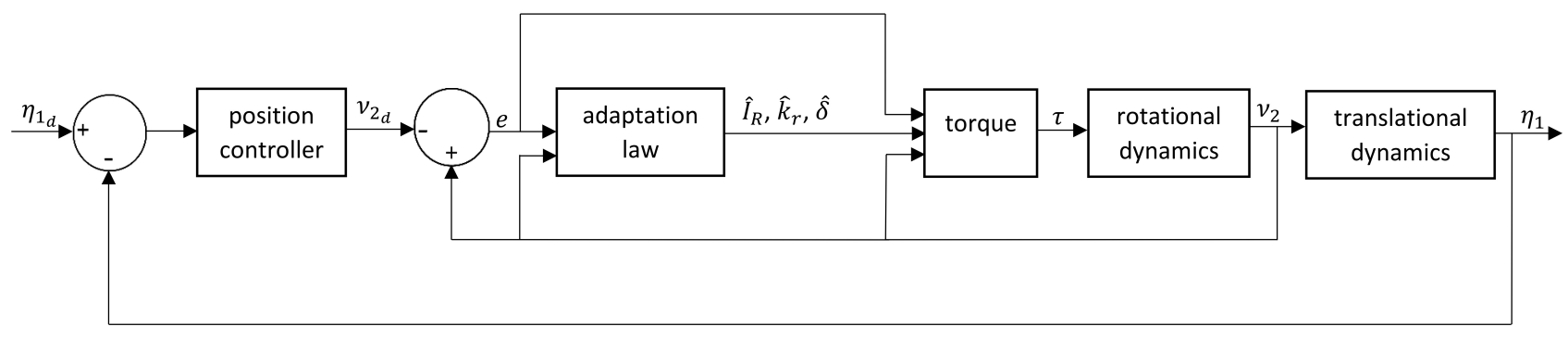

Fig. 1: The control system scheme of 6 -DOF of UAV

for some $\alpha, \gamma_{1}, \gamma_{2}, \gamma_{3}>0$ and $\tilde{\nu}_{2}=\nu_{2}-\hat{\nu}_{2}$. Then the timederivative of

$$
V\left(e, \tilde{I}_{R}, \tilde{k}_{r}, \tilde{\delta}\right)=\frac{1}{2} e^{\top} e+\frac{1}{2 \gamma_{1}} \tilde{I}_{R}^{2}+\frac{1}{2 \gamma_{2}} \tilde{k}_{r}^{2}+\frac{1}{2 \gamma_{3}} \tilde{\delta}^{2},
$$

along the closed-loop system $(8)+(19)+(20)$ is

$$
\dot{V}\left(e, \tilde{I}_{R}, \tilde{k}_{r}, \tilde{\delta}\right)=-\alpha\|e\|^{2} .
$$

Proof: The dynamics error of closed-loop system $(8)+(19)+(20)$ can be written as

$$
\begin{aligned}
\dot{e}= & -\alpha e-g_{1}\left(\nu_{2}\right)\left(\hat{I}_{R}-I_{R}\right)-g_{2}\left(\nu_{2}\right)\left(\hat{k}_{r}-k_{r}\right) \\
& -h\left(\nu_{2}\right)(\hat{\delta}-\delta) .
\end{aligned}
$$

Direct calculation shows that the time-derivative of $V\left(e, \tilde{I}_{R}, \tilde{k}_{r}, \tilde{\delta}\right)$ is

$$
\begin{aligned}
\dot{V}\left(e, \tilde{I}_{R}, \tilde{k}_{r}, \tilde{\delta}\right)= & e^{\top} \dot{e}+\frac{1}{\gamma_{1}} \dot{\hat{I}}_{R}\left(\hat{I}_{R}-I_{R}\right) \\
& +\frac{1}{\gamma_{2}} \dot{\hat{k}}_{r}\left(\hat{k}_{r}-k_{r}\right)+\frac{1}{\gamma_{3}} \dot{\hat{\delta}}(\hat{\delta}-\delta) \\
= & e^{\top}\left(-\alpha e-g_{1}\left(\nu_{2}\right)\left(\hat{I}_{R}-I_{R}\right)\right. \\
& \left.-g_{2}\left(\nu_{2}\right)\left(\hat{k}_{r}-k_{r}\right)-h\left(\nu_{2}\right)(\hat{\delta}-\delta)\right) \\
& +e^{\top} g_{1}\left(\nu_{2}\right)\left(\hat{I}_{R}-I_{R}\right) \\
& +e^{\top} g_{2}\left(\nu_{2}\right)\left(\hat{k}_{r}-k_{r}\right)+e^{\top} h(\hat{\delta}-\delta) \\
= & -\alpha\|e\|^{2}-e^{\top}\left(g_{1}\left(\nu_{2}\right)\left(\hat{I}_{R}-I_{R}\right)\right. \\
& \left.+g_{2}\left(\nu_{2}\right)\left(\hat{k}_{r}-k_{r}\right)+h\left(\nu_{2}\right)(\hat{\delta}-\delta)\right) \\
& +e^{\top}\left(g_{1}\left(\nu_{2}\right)\left(\hat{I}_{R}-I_{R}\right)\right. \\
& \left.+g_{2}\left(\nu_{2}\right)\left(\hat{k}_{r}-k_{r}\right)+h(\hat{\delta}-\delta)\right) \\
= & -\alpha\|e\|^{2} .
\end{aligned}
$$

From the dynamics (8) under controllers (19) and (20), we can see that $e(t), \tilde{I}_{R}, \tilde{k}_{r}$, and $\tilde{\delta}$ are bounded. By Barbalat's Lemma as presented in [4], we can conclude that $\lim _{t \rightarrow \infty} e(t)=0$. This completes the proof.

For a better presentation, we present a fully control scheme of UAV as illustrated in Figure 1. From the figure we can see that outer-loop contains a control scheme for tracking control position and inner-loop contains an adaptive scheme for rotational dynamics.

\section{Simulation Results}

In this section, we conduct a simulation to demonstrate the effectiveness of our approach. For translational dynamics, translational controller in Section III-A is proposed with $K_{P}=$ $K_{D}=1$. Meanwhile, the attitude dynamics is maintained by the controller in Theorem 3.1. The gain control protocol (19) and adaptive law (20) are selected as below

$$
\begin{aligned}
\alpha & =10000, \gamma_{1}=10000 \\
\gamma_{2} & =100, \gamma_{3}=2000 .
\end{aligned}
$$

In this simulation setting, an external disturbance $h=$ $\left[\begin{array}{lll}\sin (t) & 0.8 \sin (t) & 0.6 \sin (t)\end{array}\right]^{\top}$ is added with an unknown constant $\delta=0.2$.

The parameters of quadrotor UAV used in this simulation is presented in Table I [7]

TABLE I: The parameters of a quadrotor UAV

\begin{tabular}{|c|c|c|}
\hline Parameter name & Notation & Value \\
\hline Mass & $m$ & $0.52 \mathrm{~kg}$ \\
Gravity acceleration & $g$ & $9.8 \mathrm{~m} / \mathrm{s}^{2}$ \\
Translational drag coefficient & $k_{t}$ & 0.95 \\
Rotational drag coefficient & $k_{r}$ & 0.105 \\
Arm length & $l$ & $0.205 \mathrm{~m}$ \\
Drag factor & $d$ & $7.5 e^{-7} \mathrm{~kg} \cdot \mathrm{m}^{2} \mathrm{~g}$ \\
Propeller inertia & $I_{R}$ & $3.36 e^{-5} \mathrm{~kg} \cdot \mathrm{m}^{2}$ \\
Inertia of $x$-axis & $I_{x}$ & $0.0069 \mathrm{~kg} \cdot \mathrm{m}^{2}$ \\
Inertia of $y$-axis & $I_{y}$ & $0.0069 \mathrm{~kg} \cdot \mathrm{m}^{2}$ \\
Inertia of $z$-axis & $I_{z}$ & $0.0129 \mathrm{~kg} \cdot \mathrm{m}^{2}$ \\
\hline
\end{tabular}

The simulation results of the translational controller and classical adaptive approach can be seen in Figures 2-8. From the simulation results, we can see that a virtual control input using PD controller can maintain the tracking control position of UAV. Also, we can see the effectiveness of our adaptive approach to handle uncertain parameters. Therefore the attitude angles $\phi, \theta$, and $\psi$ can be stabilized. These results show that the performance of our control approach to successfully maintain 6-DOF of UAV, as concluded in Section III-A and Theorem 3.1. 

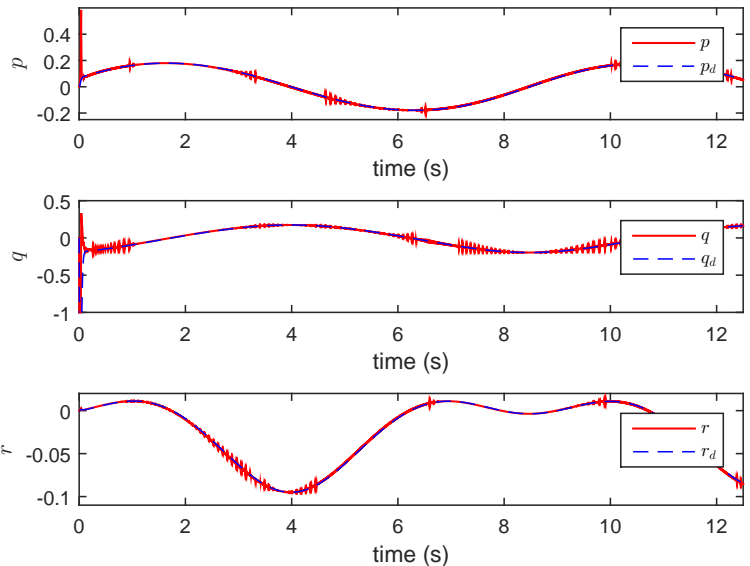

Fig. 2: Profile of $p, q$ and $r$


Fig. 3: Profile of $\phi, \theta$ and $\psi$
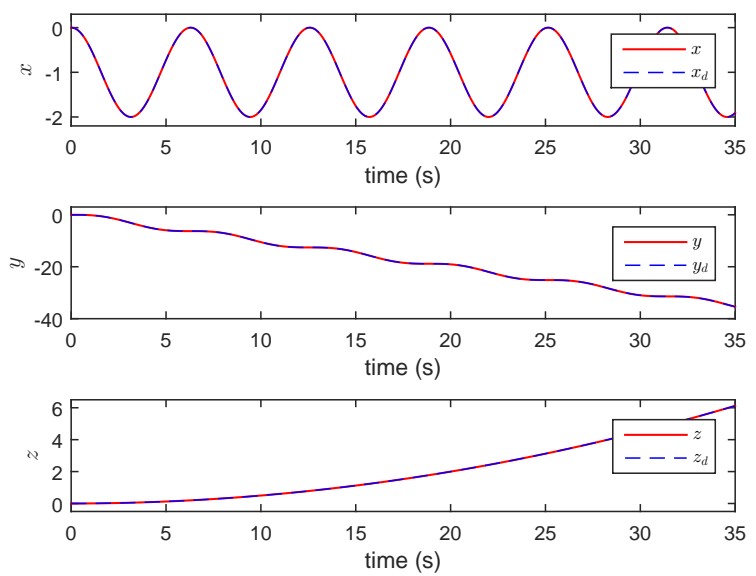

Fig. 4: Profile of $x, y$ and $z$
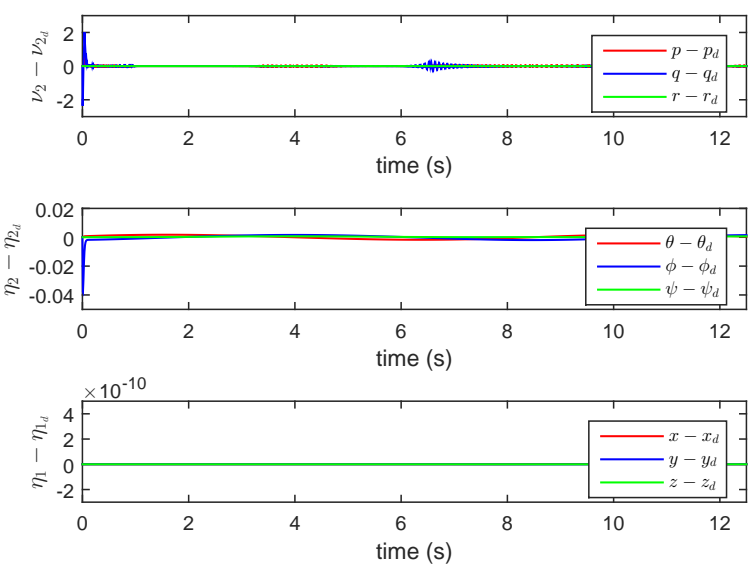

Fig. 5: Profile of tracking error trajectories

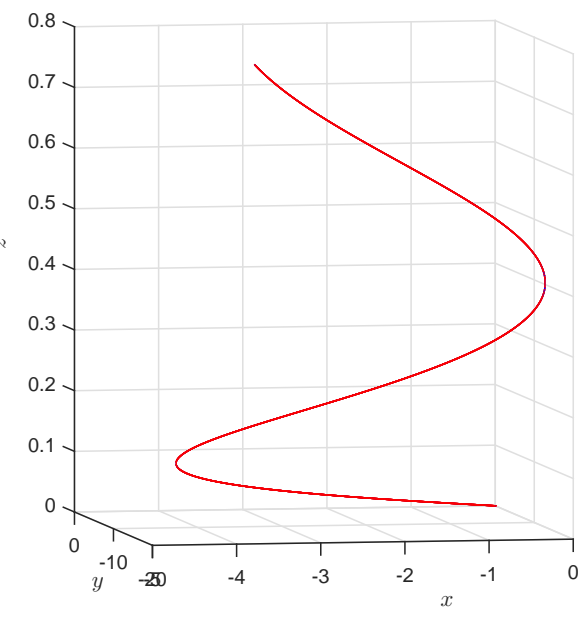

Fig. 6: Profile of $x, y$ and $z$


Fig. 7: Profile of $\tau$ 

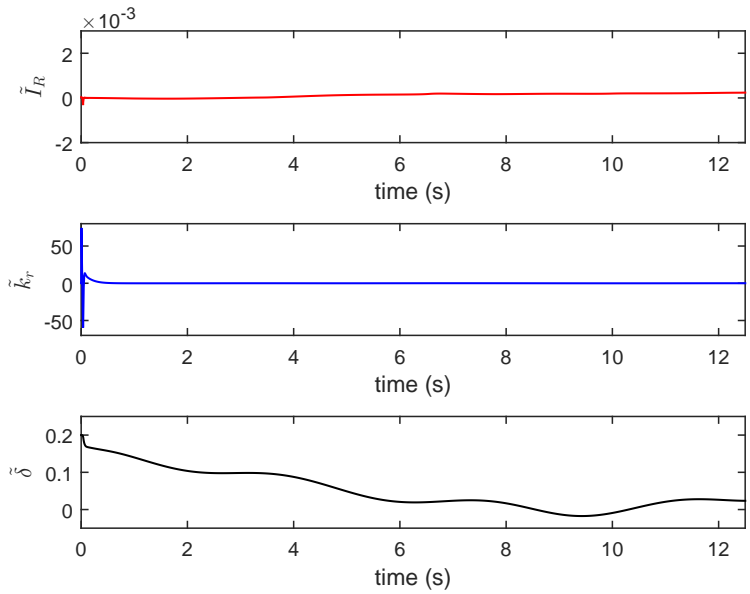

Fig. 8: Profile of estimation error

\section{CONCLUSION AND DIRECTIONS FOR FUTURE WORK}

A fully tracking control for 6-DOF of UAV with uncertain parameters is presented in this paper. We propose a virtual control input using PD controller for tracking control design. An adaptive controller is designed for tracking control of rotational dynamics. Some parameters in the nonlinear dynamics such as propeller inertia, translational drag and an external disturbance parameter are unknown for feedback control design. We develop an adaptive scheme using the certainty equivalence principle to handle the uncertainties. By applying Barbalat's Lemma, we can conclude that the rotational states can follow the desired trajectories. We also present a simulation for a drone to see the effectiveness of our approaches. It will be interesting to extend this scheme for a more sophisticated controller with a simple structure and apply it in the practical implementation.

\section{REFERENCES}

[1] Brian DO Anderson et al. Failures of adaptive control theory and their resolution. Communications in Information \& Systems, 5(1):1-20, 2005.

[2] Alessandro Astolfi, Dimitrios Karagiannis, and Romeo Ortega. Nonlinear and adaptive control with applications. Springer Science \& Business Media, 2007.

[3] Sami Elferik and Imil Hamda Imran. Control of nonholonomic mobile robot based on immersion and invariance adaptive control. In 2015 IEEE 12th International MultiConference on Systems, Signals \& Devices (SSD15), pages 1-5. IEEE, 2015.

[4] Naira Hovakimyan and Chengyu Cao. L1 adaptive control theory: guaranteed robustness with fast adaptation, volume 21. SIAM-Society for Industrial and Applied Mathematics, 2010.

[5] Imil Hamda Imran. Cooperative control of heterogeneous systems based on immersion and invariance adaptive control. Master's thesis, King Fahd University of Petroleum and Minerals, KSA, 2015.
[6] Imil Hamda Imran and Allahyar Montazeri. Adaptive control of quadrotor unmanned aerial vehicle with uncertainties. 2020 (submitted).

[7] Arief Barkah Koesdwiady. Immersion and invariance control design for unmanned aerial vehicle. Master's thesis, King Fahd University of Petroleum and Minerals (Saudi Arabia), 2013.

[8] Xiangbin Liu, Romeo Ortega, Hongye Su, and Jian Chu. Immersion and invariance adaptive control of nonlinearly parameterized nonlinear systems. IEEE Transactions on Automatic Control, 55(9):2209-2214, 2010.

[9] Allahyar Montazeri and Josef Ekotuyo. Development of dynamic model of a 7dof hydraulically actuated teleoperated robot for decommissioning applications. In 2016 American Control Conference (ACC), pages 12091214. IEEE, 2016.

[10] Allahyar Montazeri, Aydin Can, and Imil Hamda Imran. Unmanned aerial systems: Autonomy, cognition and control. In Unmanned aerial systems: theoretical foundation and applications. Elsevier, 2020 (accepted).

[11] Kumpati S Narendra and Anuradha M Annaswamy. Stable adaptive systems. Prentice Hall: Englewood Cliffs. NJ, 1989.

[12] Hamidreza Nemati and Allahyar Montazeri. Analysis and design of a multi-channel time-varying sliding mode controller and its application in unmanned aerial vehicles. IFAC-PapersOnLine, 51(22):244-249, 2018.

[13] Hamidreza Nemati and Allahyar Montazeri. Design and development of a novel controller for robust attitude stabilisation of an unmanned air vehicle for nuclear environments. In 2018 UKACC 12th International Conference on Control (CONTROL), pages 373-378. IEEE, 2018.

[14] Hamidreza Nemati and Allahyar Montazeri. Output feedback sliding mode control of quadcopter using imu navigation. In 2019 IEEE International Conference on Mechatronics (ICM), volume 1, pages 634-639. IEEE, 2019.

[15] Abdul-Wahid A Saif and Imil H Imran. Control of yaw motion of nonlinear unmanned underwater vehicle over wireless network. In 2017 14th International MultiConference on Systems, Signals \& Devices (SSD), pages 823-826. IEEE, 2017.

[16] Holger Voos. Nonlinear control of a quadrotor micro-uav using feedback-linearization. In 2009 IEEE International Conference on Mechatronics, pages 1-6. IEEE, 2009.

[17] Jing-Jing Xiong and Guobao Zhang. Discrete-time sliding mode control for a quadrotor uav. Optik, 127(8): 3718-3722, 2016.

[18] Qing-Li Zhou, Youmin Zhang, Camille-Alain Rabbath, and Didier Theilliol. Design of feedback linearization control and reconfigurable control allocation with application to a quadrotor uav. In 2010 Conference on Control and Fault-Tolerant Systems (SysTol), pages 371376. IEEE, 2010. 